\title{
BMJ Open Close look at the experiences of patients enrolled in a clinical trial of acupuncture treatment for atrial fibrillation in Korea: a qualitative study nested within a randomised controlled trial
}

\author{
Seung Min Kathy Lee, ${ }^{1}$ Jungtae Leem, ${ }^{1}$ Jun Hyeong Park, ${ }^{2}$ Kang Hyun Yoon, ${ }^{2}$ \\ Jong Shin Woo, ${ }^{3}$ Jung Myung Lee, ${ }^{3}$ Jin-Bae Kim, ${ }^{3}$ Weon Kim, ${ }^{3}$ Sanghoon Lee ${ }^{1}$
}

To cite: Lee SMK, Leem J, Park JH, et al. Close look at the experiences of patients enrolled in a clinical trial of acupuncture treatment for atrial fibrillation in Korea: a qualitative study nested within a randomised controlled trial. BMJ Open 2017;7:e013180. doi:10.1136/bmjopen-2016013180

- Prepublication history and additional material is available. To view please visit the journal (http://dx.doi.org/ 10.1136/bmjopen-2016013180).

Received 24 June 2016 Revised 28 December 2016 Accepted 13 January 2017

CrossMark

For numbered affiliations see end of article.

Correspondence to Dr Sanghoon Lee; shlee777@gmail.com

\section{ABSTRACT}

Objective: To explore the experiences of patients with atrial fibrillation (AF) in the context of a prospective, two-parallel-armed, participant-blinded and assessorblinded sham-controlled randomised trial.

Design: A nested qualitative study within an ongoing randomised controlled trial to explore acupuncture's antiarrhythmic effects on drug refractory acupuncture in persistent atrial fibrillation (AF) (ACU-AF trial). Participants: Participants were recruited using purposeful sampling and a maximum variation strategy with regard to treatment allocation (treatment or control) and protocol completion (completion or non-completion).

Setting: This was a single-centre in-depth interview qualitative study conducted at a tertiary-level university hospital in Seoul, Republic of Korea.

Results: Data saturation was reached after 8 participants. Thematic analysis identified that most patients were not aware of their condition until medical check-up; physician referral was the main reason for trial participation, and patients had high expectations regardless of previous acupuncture experiences. Patients tended to depend on their physicians' opinions because they felt helpless of their condition. No one questioned their assigned treatment groups and generally believed acupuncture treatment was different for cardiovascular diseases. A few patients expressed disappointment in the strict and rigid protocols, in which most practitioners refrained from explaining their acupuncture procedures.

Conclusions: For cardiovascular patients their physician's advice was one of the biggest reasons for enrolling in the acupuncture trial therefore relying on standard recruitment methods may not be effective. Fortunately both real and sham acupuncture groups in our sample were receiving treatment as intended, but in the future, designing a more pragmatic trial (better reflecting clinical settings, expanding the inclusion criteria and using more treatment points) will allow researchers to better explore the comprehensive effects of acupuncture. The findings of this study will allow researchers to improve the currently ongoing ACU-AF

\section{Strengths and limitations of this study}

- This was a qualitative study conducted within an ongoing main trial, allowing researchers to keep patient data and save time and costs, while concurrently exploring areas for improvement.

- Patients were selected to include a variety of experiences from the control group, treatment group, those who completed the trial and those who dropped out.

- Several areas for improvement were identified and a few apprehensions that researchers had when conducting the trial were resolved.

- Although information saturation was accomplished; we interviewed only a small number of participants and most were residents living in the hospital vicinity, which may limit the external validity of our findings.

trial and to further help interpretation of main trial outcomes once the trial is completed.

Trial registration number: NCT02110537.

\section{BACKGROUND}

Atrial fibrillation (AF) is an increasing physical, psychological and economic health burden worldwide affecting $\sim 2 \%$ of people in Europe and North America ${ }^{1}{ }^{2}$ and 1.7\% in Korea. ${ }^{3}$ It is an independent risk factor of stroke, associated with several chronic diseases $^{4}$ and related to significantly lower quality of life in patients compared with other general diseases. ${ }^{5}$ Furthermore, the prevalence rates for AF increase rapidly with age meaning that countries such as Korea and Japan with one of the fastest ageing populations $^{6}$ are most likely to experience significant repercussions. 
Among complementary and alternative treatments, acupuncture is a widely used therapy that has already been investigated for the treatment of a variety of cardiac arrhythmias, including paroxysmal supraventricular tachycardia, ${ }^{7} 8$ ventricular premature beat and $\mathrm{AF}^{9}{ }^{10}$ The less invasive nature of acupuncture compared with other therapies and fewer side effects makes it an intriguing therapeutic option; however, most published randomised controlled trials (RCTs) related to acupuncture and arrhythmia to date are of poor methodological quality and insufficient to accurately evaluate its usefulness. ${ }^{11}$

In 2011, a clinical trial using acupuncture to reduce recurrence rates in patients with $\mathrm{AF}$ after electrical cardioversion (EC) found that patients in the sham and no treatment group were 2.77 times more likely to experience $\mathrm{AF}$ recurrence compared with those that received acupuncture, ${ }^{10}$ although the trial was affected by limitations. We attempted to reproduce the previous trial and provide further insights into the therapeutic mechanisms underlying acupuncture by using only acupuncture points that have been more extensively researched in other preclinical trials such as PC5, PC6, ST36 and ST37, as well as electroacupuncture. ${ }^{12} 13$ However, while conducting our trial, questions were raised regarding ways to increase patient recruitment, to verify the validity of interventions and to further improve the trial design if needed. With few high-quality trials to refer to, nesting a qualitative study within the early stages of the main trial was suggested.

Nesting qualitative studies within an existing trial allows researchers to explore meaningful but indiscrete areas within the main quantitative trial, identify reasons for poor recruitment, understand complex interventions and analyse null trial results. ${ }^{14}$ Such studies can be conducted prior, during or at the end of the trial.

Since we wanted to explore patient experiences within a time frame in which researchers and participants could recollect as much detail as possible, our qualitative study was conducted during an early stage of the ongoing trial, but as a separate unit and avoiding a time point that could affect the main outcome. Conducting the study concurrently with the main trial allows researchers to incorporate the information learnt into the trial right away, while also saving patient data, time and related costs. The main purpose of this study was to explore what eventually propelled the participants to enrol into the trial, what they expected and experienced from the trial, whether the real and sham acupuncture treatments were received as intended, and whether the trial outcome measures were accurately reflecting patient needs.

\section{METHODS}

This qualitative study was a substudy nested within a currently ongoing RCT (ACU-AF). Details of the trial protocol have been published earlier. ${ }^{15}$ In order to investigate the experiences of patients going through $\mathrm{AF}$ during the trial and treatment, and ultimately to explore areas for improvement, in-depth interviews with telephone follow-up were conducted. For triangulation, interview transcripts, notes taken during the interviews and previous medical records were also searched but the primary sources of investigation were patient interviews. Other interventions and long-term researcher involvement were not necessary.

\section{Setting}

This study was a single-centre study conducted at Kyung Hee University Hospital, Seoul, Republic of Korea.

\section{Participants}

A clinical research coordinator who was in charge of scheduling patient treatments had legitimate access to all of the contact information, and thereby informed eligible participants about the study either directly during one of their prescheduled visits or by telephone. If the patient expressed interest, a general physician who was not acquainted with the patients was called in to provide further details and to obtain final consent. For those who further consented to share their experiences, interviews were scheduled on a date and time of the participants' choice.

Participants were purposefully selected using nonrandom maximum variation strategy and theoretical sampling to incorporate a variety of experiences from different groups of people including those allocated to the real acupuncture group, those allocated to the placebo acupuncture group, those who dropped-out due to cardiac events or other reasons, and those who successfully completed the trial. Owing to the nature of AF occurrences and the tertiary university hospital setting, there were a few limitations in recruiting participants from different age ranges.

Our qualitative study was focused on exploring the particular experiences of patients who were participants in an acupuncture trial. Therefore, those who declined participation in the main ACU-AF trial were automatically excluded and interviews were conducted only for patients who had received at least one treatment session of real acupuncture or placebo acupuncture. The surveyed patients did not have to complete the main trial.

\section{Data collection}

\section{Interviews}

Semistructured open-ended interviews were conducted in Korean, from June to September 2015, in a quiet and comfortable room inside the hospital either individually or accompanied by a family member. They were conducted face-to-face, recorded on tape, transcribed verbatim by two trained research assistants and rechecked by the interviewer (SMKL). A prearranged topic guide was used to direct patients back to relevant issues 
if their answers strayed from the topic (see online supplementary appendix 1). After each interview, the tape recorder was turned off and several of the main open-ended questions were repeated. In previous studies, we had frequently noticed patients providing important information after the recorder was turned off, and therefore noteworthy responses were taken down on paper as parallel notes. Within 24 hours of interviews, patients were called by phone and asked whether they had any additional information to provide. After eight interviews, no further themes emerged.

\section{Interviewer background}

The interviews were conducted by the primary author (SMKL) who is a Doctor of Traditional Korean Medicine (TKM) as designated by the Korean Minister of Health and Welfare. As a licenced medical specialist in acupuncture, she has conducted several previous qualitative studies, all related to exploring patient acceptance of and experiences with traditional and complementary medicine. She performed acupuncture treatment for two patients at the start of the ACU-AF trial and therefore those participants were excluded. The eight interviewees had no previous clinical contact with or information regarding the primary author.

\section{Data analysis}

Data were analysed by the main interviewer according to the principles of inductive thematic analysis. ${ }^{16}$ Themes were identified and grouped into categories and subcategories. Transcripts were re-read several times to capture all emerging themes and to extract supporting excerpts. Checking for intercoder reliability by an external researcher was thought to increase validity, but there have been controversies regarding this process. ${ }^{17}$ Instead, the main analysis was conducted by SMKL, and another author (KHY) reviewed and verified the results. The role of KHY was not to conduct the analysis but to review the entire analytic process of coding and to verify that there was no researcher bias or misconceptions in interpretation. For respondent validation, interim results were presented to one participant who had academic experience in conducting qualitative studies. The purpose was to receive detailed feedback on the accuracy of the analysis in portraying participant experiences. Any identifiable information about patients in the transcriptions was removed, and patients were identified only by letters and numbers throughout the transcripts. Coding was performed without using software.

After analysis, all of the transcripts were translated into English by two bilingual researchers. Where meanings were ambiguous or the particular nuance of the speaker could be lost in translation, the interviewer selected the translation that better reflected the participant's words.

This study has been reported following the Consolidated Criteria for Reporting Qualitative Research
(COREQ) checklist $^{18}$ (see online supplementary appendix 2).

\section{RESULTS}

\section{Participants}

At the time of recruitment (from May to August 2015), there were a total of 24 participants enrolled in the main ACU-AF trial. Thirteen were contacted for qualitative interviews as the interviews were being analysed. A total of five participants did not answer the phone or declined because they were busy. Recruitment ended after interviewing the eighth participant, and two researchers (SMKL and KHY) reached a consensus that saturation had been met. Patient ages ranged from 57 to 74 years (mean \pm SD, $68 \pm 6.3$ years; table 1 ).

Patient experiences were categorised as: (1) being diagnosed with AF; (2) participating in the ACU-AF trial and (3) receiving acupuncture. Their experiences while participating in the ACU-AF trial were further categorised as: (1) reasons for participating in the ACU-AF trial; (2) expectations and overall experiences with the trial; and (3) differences between real-life clinical settings and the trial. Experiences in receiving acupuncture also further revealed that participants were unable to tell if they had been assigned to the control group or the sham acupuncture group.

\section{Participant diagnosis with AF}

All of the patients received their first AF diagnosis during routine medical examinations. Immediate treatment was not sought because most patients did not have AF-related symptoms or did not recognise their symptoms as AF. Among the participants, only one knew that the perceived palpitations and chest discomfort were related to $\mathrm{AF}$.

Table 1 Participant information $(n=8)$

Number of patients with symptomatic atrial fibrillation

Number of patients without symptoms

Factors that motivated patients to participate

Doctor referral

Positive perception of acupuncture

Number of patients with high expectations of the trial

Number of patients with prior acupuncture experience

Number of patients with prior electroacupuncture

experience

Reason for previous acupuncture treatment

Low back pain

Hip pain

Knee pain

Ankle sprain

Others (gout, etc)

Number of patients who thought 10 sessions were too many

Number of patients who questioned their treatment Number of patients who would recommend the trial to others 
At first (first diagnosed in 2012), I really didn't care much about my AF. When I visited the hospital for my next checkup, I decided to ask one of the ladies at the front desk what I should do to treat my AF and hyperlipidemia. She told me that I have to go to a university hospital, which is why I decided to visit. Even then, I only first came here to get medication for my mom. (S002, M)

\section{Participating in the ACU-AF trial}

Reasons for participating: doctor referral, fear of conventional treatment, and free treatment and check-ups

All of the patients reported that they enrolled in the ACU-AF trial because they were informed about it by their physician. Since heart diseases may develop into life-altering problems, the patients were highly dependent on their cardiologists for decision-making, and there was a dominating sense of helplessness that arose from their belief that heart disease can only be accurately diagnosed and treated by a doctor and that the patient could do nothing to improve their health.

I wouldn't have participated in this trial if my doctor hadn't told me about it...The two doctors (conventional medicine and traditional medicine) must have surely started this (study) because they believed it would work. They wouldn't have started it if they thought it wouldn't work, would they? (S003, M)

Aside from doctors informing their patients about the trial, a few patients chose to participate in the ACU-AF trial to avoid receiving additional conventional treatment. They expressed fear of surgery and wished to avoid medication. Acupuncture was a new and different option that they believed to have fewer side effects. Positive preconceptions of acupuncture and the fact that acupuncture treatments were provided for free also gave them a reason to enrol. Most of the patients were retired, and depended on their savings or children for medical care.

Because taking medicine for a long period of time is not good for you. If I get acupuncture, I won't have to take any medications. Even if I do take pills, it's not going to be good for me in the long run. But with acupuncture you don't have any of this (problem). (S005, M)

\section{Expectations of the ACU-AF trial}

High expectations of acupuncture in the ACU-AF trial despite positive and negative experiences with acupuncture in the past

The patients had high expectations of the ACU-AF trial that were not related to previous positive or negative experiences with acupuncture. Six participants out of eight had undergone acupuncture in the past for a variety of reasons including low back pain, ankle sprain, knee pain, gout and hip pain. Four had positive results and two did not. The remaining two acupuncture-naive participants showed no particular affinity for acupuncture. However, most expressed high expectations related to ACU-AF. Receiving acupuncture during the trial had given them hope and provided emotional support. One patient rationalised that receiving acupuncture was a reasonable and logical solution because acupuncture is thought to promote circulation of blood and Qi.

\section{Overall experience of the ACU-AF trial}

Negative experiences of the trial for patients without AF symptoms

Most of the patients did not display AF-related symptoms when they enrolled in the trial which made it difficult for them to assess whether the trial was a positive experience for them or not. Up to one-third of patients with $\mathrm{AF}$ are known to have asymptomatic AF, which is most common in the elderly. ${ }^{19}$ Without physically tangible results, these patients did not feel that the trial was worthy of recommendation to others.

But since I didn't have any (symptoms) in particular. Like I said earlier, if I had done this when I had any symptoms to start out with, then I would be like 'oh, this treatment is really making me better'. But I first came without any of that (symptoms)...It just felt like it wasn't working because I didn't have any changes...Before I knew about my AF, I didn't feel any different and I still don't. (S003, M)

\section{Positive experiences for patients with AF symptoms and recognisable physical changes}

Only three participants displayed AF-related symptoms. One participant had frequent palpitations and shortness of breath, while the other two had milder symptoms that they had not been initially recognised. Those with mild symptoms experienced noticeable physical changes during and after the treatment, which led to an overall positive experience with the trial.

Acupuncture? Well of course it was considerably good. It was my first time but it made me feel comfortable... Symptom-wise also, I decided to try to an aggressive exercise. Not extremely aggressive, but like walking much faster than usual. I felt that it was much better than before. Specifically, I felt that my shortness of breath was much better. $(\mathrm{S} 004, \mathrm{M})$

\section{Good patient compliance with frequency and duration}

\section{of treatment sessions and medical check-ups}

Despite our initial concerns, coming to the medical centre for acupuncture once a week for 10 weeks was not burdensome for patients. Frequent examinations and surveys were also not problematic. Most participants were retired, lived near the hospital, had a strong desire to improve their health and hoped that acupuncture would help. Since their doctors had told them to come every week, they believed that this was necessary and replied that they would come more frequently if their physician told them to. One participant responded that feeling a responsibility to finish the trial was another motivation that made them follow the strict protocol. 
If by doing this, I can get better. And if the gentleman (doctor) can use this (knowledge) to treat another patient, I felt that I would be providing a lot of help. That's why I willingly decided to enrol in this trial. This is why I finished all of the 10 sessions. If he told me to come 20 times then I would have also come 20 times. ... Even if I had to come every day I would have. My house is very close anyway. (S003, M)

\section{Differences between real-life clinical settings and the trial, and what patients wanted from the trial}

A negative aspect of the trial was lack of sufficient explanation and discussions with the doctors while receiving acupuncture treatment. Many of the patients reported that unlike other clinics, where the acupuncturist communicates actively with the patient throughout the sessions, they felt that the doctors at this hospital were more stoic. The ambience of the treatment environment was less inviting compared with those of other local clinics.

They wouldn't explain. After check up, (they provide) treatment. After treatment, (they give) intradermal acupuncture (for self application). I was expecting them to tell me how that (acupuncture) treatment would make what part (of my body) better. (But) what more was there during the trial than them (acupuncturists) coming in and out to insert and pull out the needles? (S005, M)

\section{Experience receiving acupuncture}

Participants were unable to determine their group assignment

Six participants had prior experience with acupuncture but none had received it for heart-related diseases and among the six, only two had received electroacupuncture. Of the two electroacupuncture-experienced participants, one had been randomised to the sham control group, in which the needle was placed on a nonacupuncture point without any electrical conduction. The participant noticed that the treatment was weaker, but did not question the validity of the treatment nor the treatment group. Overall, there was a tendency among sham control participants to dismiss differences because they believed that acupuncture for heart disease was different from treatment for other conditions.

\section{DISCUSSION}

Main findings

This study was the first to explore the acupuncture trial experiences of patients with $\mathrm{AF}$ while a main trial was ongoing, and revealed several important factors related to AF and the ACU-AF trial in which the study was conducted. These factors will be crucial to reflect on after the main trial has been completed.

For the participants in this study, conventional treatment was not merely an option but a priority, and acupuncture was an alternative add-on treatment that was sought with their cardiologists' consent and support. Since there was a gap from diagnosis to initial AF treatment, patients were willing to comply with and commit to physician orders with a sense of urgency to compensate for the loss of time. This feeling also led to a heightened dependency on physician opinions. Thus, to increase patient recruitment, providing information about the trial to other cardiologists may be a more feasible option than using standard recruitment methods. To avoid inadvertent coercion of participants by healthcare practitioners, physicians must avoid mentioning that the study will be beneficial and rather focus on informing patients of a currently ongoing trial that they may be eligible for participation. It will also be necessary to seek cooperation from cardiologists that are not affiliated with the trial and able to provide them with an objective perspective.

Many of the patients in our trial did not have AF-related symptoms. In the ACU-AF trial, distinguishing patients with symptomatic and asymptomatic $\mathrm{AF}$ in the inclusion criteria or including more patients with symptoms would help researchers further interpret acupuncture effects in relation to their physical changes. Symptomatic AF may be a more accurate diagnosis criterion, especially for acupuncture trials, since treatment points are primarily selected based on patient symptoms rather than the pathological diagnosis itself.

In interviews, the patients frequently expressed a need for emotional relief. This finding is important because it provides a previously unforeseen interpretation of acupuncture effects reported in previous trials and also a future area for research. Several studies have reported a relationship between AF recurrence after EC and depression, and that depression is the only independent predictor of $\mathrm{AF}$ recurrence. ${ }^{20}$ Based on a literature review of previous preclinical research, the points used in our trial (PC5, PC6, ST36 and ST37) are known to inhibit cardiac sympathetic-related centres in the central nervous system through type III and IV sensory nerve fibres located on the median and deep peroneal nerves. ${ }^{14}$ However, a previous trial by Lomuscio and colleagues used different points (HT7, PC6 and BL15). Although little preclinical research exists on HT7, in the clinic it is considered an important treatment point for relieving emotional and psychological issues. If at the end of the ACU-AF trial we identify differences in treatment effects from those observed by Lomuscio and colleagues, more research into the differences between acupuncture points would be warranted. Since recent practice-based effectiveness research has also reported that the adjunctive use of integrative medicine is able to decrease pain and anxiety levels in cardiovascular patients ${ }^{21}$ focusing future treatments on relieving symptoms of psychological distress may be a feasible approach.

The patients in our study showed no signs of allocation exposure and even among those with abundant acupuncture experience; no one believed that they had 
received sham treatment. In studies that use complex healthcare interventions such as acupuncture, blinding patients and successfully maintaining these controls throughout the trial is often a challenge. The successful maintenance of blinding in our trial can be attributed to the patients' lack of experience with acupuncture for treating AF.

The most noteworthy difference between the ACU-AF trial participants and those who had enrolled in previous acupuncture trials were reasons for participation (table 2). Previously, our research group had conducted a qualitative study of patients with Bell's palsy to explore their experiences and reasons for seeking acupuncture. ${ }^{22}$ Among patients with Bell's palsy, dissatisfaction with conventional medicine was the primary motive for seeking acupuncture. Although such patients go through extreme distress from the loss of facial muscle control and conspicuous facial disfigurement, the lack of treatment options other than steroids during the acute stages of the disease causes great dissatisfaction and a strong desire for a more concentrated healthcare programme. Dissatisfaction was the primary motivation for choosing acupuncture among chronic fatigue patients as well. ${ }^{23}$ Those who participated in an acupuncture trial for low back pain noted distrust of surgery or drugs as their primary reasons for participation in the acupuncture trial. ${ }^{24}$ Such differences between conditions emphasise the need for using different approaches to integrate acupuncture in clinical settings.

Our interviews revealed that there was a lack of necessary education programmes regarding $\mathrm{AF}$ and a lack of follow-up consultations after medical examinations were completed. Therefore, education programmes should immediately be incorporated into clinic and hospital settings to help patients start treatment earlier and to maintain a more active attitude towards AF.

\section{Strengths and limitations}

Our study results are restricted to patients who enrolled in the ACU-AF trial being conducted in a single medical centre in Seoul, Republic of Korea. Most patients who consented to the interview were over 60 years old, retired, of low educational status and lived in the vicinity of the medical centre. In addition, despite purposeful selection, our study participants were mostly men, and we did not assess the experiences of those who refused to participate. Those participants who were asymptomatic did not have much experiences to share related to the acupuncture treatment and much of the discussion was limited to trial experiences only. Even if they had prior experience of receiving acupuncture treatment, a detailed comparison was challenging because most had received it more than 3 months ago (as was the inclusion criterion of the main trial). We believe that such homogeneity of asymptomatic participants and narrowing of discussion topic may have contributed to our study reaching data saturation with only eight participants. The treatments and check-ups were all provided for free, which may mean that the patient experiences may not adequately reflect those in real clinical contexts. However, since AF occurs more frequently in men and acupuncture in Korea is not costly (generally $<\$ 10$ per session for elderly patients), we do not believe that including more women or charging for treatments would have changed the results.

The fact that semistructured interviews were the main source of data may be a weakness of this study. However, methods of triangulation have also received criticism, and focus group interviews would have made it difficult to schedule every patient to visit at a specified date and time. Conducting one-on-one interviews allowed for flexible scheduling of interviews, and for more honest and open conversations about the trial. In addition to the above shortcomings, our results may be affected by recall bias because the interviews were conducted on patients who had dropped out or completed the trial 19 months previously.

\section{CONCLUSIONS}

The results of the present nested qualitative study found that patients enrolled in acupuncture trials mainly through doctor referral and did not find the trial design perplexing. The control group did not question the

Table 2 Reasons for acupuncture treatment according to disease

\begin{tabular}{|c|c|c|c|}
\hline & Low back pain & Bell's palsy & Atrial fibrillation \\
\hline Reason for acupuncture & Distrust of conventional medicine & $\begin{array}{l}\text { Dissatisfaction with conventional } \\
\text { medicine }\end{array}$ & Doctor referral \\
\hline $\begin{array}{l}\text { Acupuncture } \\
\text { satisfaction rates }\end{array}$ & High & High & $\begin{array}{l}\text { Mixed responses } \\
\text { Low in asymptomatic AF }\end{array}$ \\
\hline Patient attitudes & $\begin{array}{l}\text { Actively participate and search } \\
\text { out treatment }\end{array}$ & $\begin{array}{l}\text { Actively participate and search } \\
\text { out treatment }\end{array}$ & $\begin{array}{l}\text { Extremely passive regarding } \\
\text { treatment } \\
\text { Very dependent on doctor }\end{array}$ \\
\hline $\begin{array}{l}\text { Familiarity with } \\
\text { acupuncture }\end{array}$ & Very familiar & Not familiar & Not familiar \\
\hline $\begin{array}{l}\text { Attenuation of other } \\
\text { symptoms }\end{array}$ & $\begin{array}{l}\text { Alleviated headache, fatigue, } \\
\text { improved sleep and constipation }\end{array}$ & Great emotional relief & Slight emotional relief \\
\hline
\end{tabular}


validity of the treatment, and most had high expectations of the trial. However, most patients were asymptomatic, which affected their overall satisfaction after enrolling in the trial, and the treatment environment was not very similar to that of a real clinic because patients and researchers were strictly prohibited from openly communicating. ${ }^{15}$

In the future, choosing acupuncture points aimed to relieve patient anxiety related to $\mathrm{AF}$ is warranted. To increase external validity, it may be desirable to expand the inclusion criteria of studies like this one to include more patients with symptomatic $\mathrm{AF}$, even if this requires adding patients with paroxysmal $\mathrm{AF}$ to the trial. A more pragmatic clinical trial design that would better incorporate TKM values may also be preferred.

\section{Author affiliations}

${ }^{1}$ Department of Clinical Korean Medicine, Graduate School, Kyung Hee University, Seoul, Republic of Korea

${ }^{2}$ Department of Acupuncture and Moxibustion, College of Korean Medicine, Kyung Hee University, Dongdaemun-gu, Republic of Korea

${ }^{3}$ Division of Cardiology, Department of Internal Medicine, Kyung Hee

University Hospital, Kyung Hee University, Seoul, Republic of Korea

Acknowledgements The English grammar of the manuscript was edited by eWorld Editing.

Contributors SMKL conducted the interviews and analysis, rechecked the transcribed manuscript, interpreted the data, and drafted the article.

$\mathrm{JL}$ critically reviewed the entire manuscript and provided significant feedback. JHP proofread the article and helped with data organisation. KHY helped with data analysis. JSW, JML and J-BK provided guidance drafting the article. WK and SL guided and supervised the research process as co-principal investigators.

Funding This work was supported by grants from the Traditional Korean Medicine R\&D Project, Korean Ministry of Health and Welfare (HI13C0580 and HI15C0166).

Competing interests None declared.

Patient consent Obtained.

Ethics approval The study protocol for the qualitative trial was approved by the Kyung Hee University Korean Medicine Hospital Institutional Review Board (IRB\#: 150515-HR-017).

Provenance and peer review Not commissioned; externally peer reviewed.

Data sharing statement No additional data are available.

Open Access This is an Open Access article distributed in accordance with the Creative Commons Attribution Non Commercial (CC BY-NC 4.0) license, which permits others to distribute, remix, adapt, build upon this work noncommercially, and license their derivative works on different terms, provided the original work is properly cited and the use is non-commercial. See: http:// creativecommons.org/licenses/by-nc/4.0/

\section{REFERENCES}

1. Zoni-Berisso M, Lercari F, Carazza T, et al. Epidemiology of atrial fibrillation: European perspective. Clin Epidemiol 2014;6:213-20.
2. Kirchhof $\mathrm{P}$, Auricchio $\mathrm{A}, \mathrm{Bax} \mathrm{J}$, et al. Outcome parameters for trials in atrial fibrillation: executive summary. Eur Heart $J$ 2007;28:2803-17.

3. Son MK, Lim NK, Cho MC et al. Incidence and risk factors for atrial fibrillation in Korea: the National Health Insurance Service Database (2002-2010). Korean Circ J 2016;46:e71.

4. Wolf PA, Abbott RD, Kannel WB. Atrial fibrillation as an independent risk factor for stroke: the Framingham study. Stroke 1991;22:983-8.

5. Thrall G, Lane D, Carroll D, et al. Quality of life in patients with atrial fibrillation: a systematic review. Am J Med 2006;119:5:448e1-e19.

6. United Nations, Department of Economic and Social Affairs, Population Division (2015). World Population Ageing 2015 (ST/ESA SER.A/390). 2015. http://www.un.org/en/development/desa/ population/publications/pdf/ageing/WPA2015_Report.pdf. (accessed 1 Nov 2016).

7. Dong S. 32 cases of paroxysmal supraventricular tachycardia in acupuncture Neiguan. J Henan Univ Chin Med 2006;21:69-70.

8. Wu RD, Lin LF. Clinical observation on wrist-ankle acupuncture for treatment of paroxysmal supraventricular tachycardia. Zhongguo Zhen Jiu 2006;26:854-6.

9. Lomuscio A, Belletti S, Battezzati PM, et al. Efficacy of acupuncture preventing atrial fibrillation recurrences after electrical cardioversion. J Cardiovasc Electrophysiol 2011;22:241-7.

10. Lombardi F, Belletti S, Battezzati PM, et al. Acupuncture for paroxysmal and persistent atrial fibrillation: an effective nonpharmacological tool? World J Cardiol 2012;3:60-5.

11. Kim TH, Choi TY, Lee MS, et al. Acupuncture treatment for cardiac arrhythmias: a systematic review of randomized controlled trials. Int J Cardiol 2011;149:263-5.

12. Longhurst JC. Electroacupuncture treatment of arrhythmias in myocardial ischemia. Am J Physiol Heart Circ Physiol 2007;292: $\mathrm{H} 2032-4$.

13. Li P, Tjen-A-Looi SC. Mechanism of the inhibitory effect of electroacupuncture on experimental arrhythmias. J Acupunct Meridian Stud 2013;6:69-81.

14. O'Cathain A, Thomas KJ, Drabble SJ, et al. What can qualitative research do for randomized controlled trials? A systematic mapping review. BMJ Open 2013;3:e002889.

15. Park J, Kim HY, Lee SM, et al. Acupuncture antiarrhythmic effects on drug refractory persistent atrial fibrillation: study protocol for a randomized controlled trial. Evid Based Complement Alternat Med 2015;2015:613970.

16. Braun V, Clarke V. Using thematic analysis in psychology. Qual Res Psychol 2006;3:77-101.

17. Greenhalgh T. How to read a paper: the basics of evidencebased medicine 5th edition. London: BMJ Publishing Books, 2014:167.

18. Tong A, Sainsbury P, Craig J. Consolidated criteria for reporting qualitative research (COREQ): a 32-item checklist for interviews and focus groups. Int J Qual Health Care 2007;19:349-57.

19. Camm AJ, Kirchhof P, Lip GY, et al. Guidelines for the management of atrial fibrillation: the Task Force for the Management of Atrial Fibrillation of the European Society of Cardiology (ESC). Eur Heart J 2010;31:2369-429.

20. Lange HW, Herrmann-Lingen C. Depressive symptoms predict recurrence of atrial fibrillation after cardioversion. J Psychosom Res 2007;63:509-13.

21. Johnson JR, Crespin DJ, Griffin KH, et al. The effectiveness of integrative medicine interventions on pain and anxiety in cardiovascular inpatients: a practice-based research evaluation. BMC Complement Altern Med 2014:14:486.

22. Lee SM, Yang SP, Kim ES, et al. Admission care for Bell's palsy patients: a qualitative report on patient experiences. J Kor Acup Mox 2013;30:11-23.

23. Son HM, Park EY, Kim DH, et al. Experiences with, perceptions of and attitudes towards traditional Korean medicine (TKM) in patients with chronic fatigue: a qualitative, one-on-one, in-depth interview study. BMJ Open 2015;5:e006178.

24. Kim SY, Lee KB, Lee HS, et al. Acupuncture experience in patients with chronic low back pain (2): a qualitative study-focused on participants in randomized controlled trial. J Kor Acup Mox 2012;29:581-97. 


\section{Correction}

Lee SMK, Leem J, Park JH, et al. Close look at the experiences of patients enrolled in a clinical trial of acupuncture treatment for atrial fibrillation in Korea: a qualitative study nested within a randomised controlled trial. BMJ Open 2017;7:e13180. doi: 10.1136/bmjopen-2016-013180

There should be two Corresponding authors. Both Sanghoon Lee and Weon Kim should be considered the Corresponding authors for this paper. The Correspondence section should read:

\section{Correspondence to}

Dr Sanghoon Lee; shlee777@gmail.com

and

Weon Kim; mylovekw@hanmail.net

Open Access This is an Open Access article distributed in accordance with the Creative Commons Attribution Non Commercial (CC BY-NC 4.0) license, which permits others to distribute, remix, adapt, build upon this work noncommercially, and license their derivative works on different terms, provided the original work is properly cited and the use is non-commercial. See: http://creativecommons.org/licenses/by-nc/4.0/

BMJ Open 2017;7:e013180corr1. doi:10.1136/bmjopen-2016-013180corr1 